This work is on a Creative Commons Attribution 4.0 International (CC BY 4.0) license, https://creativecommons.org/licenses/by/4.0/. Access to this work was provided by the University of Maryland, Baltimore County (UMBC) ScholarWorks@UMBC digital repository on the Maryland Shared Open Access (MD-SOAR) platform.

Please provide feedback

Please support the ScholarWorks@UMBC repository by emailing scholarworks-group@umbc.edu and telling us what having access to this work means to you and why it's important to you. Thank you. 


\title{
EGU21-2256
}

https://doi.org/10.5194/egusphere-egu21-2256

EGU General Assembly 2021

(c) Author(s) 2021. This work is distributed under

the Creative Commons Attribution 4.0 License.

\section{Improving the treatment of subgrid cloud variability in warm rain simulation in CESM2}

\author{
Hao Wang ${ }^{1}$, Minghuai Wang ${ }^{2}$, Daniel Rosenfeld ${ }^{3}$, Yannian Zhu ${ }^{4}$, and Zhibo Zhang ${ }^{5}$ \\ ${ }^{1}$ School of Atmospheric Sciences, Nanjing University, China (wang_hao@smail.nju.edu.cn) \\ ${ }^{2}$ School of Atmospheric Sciences, Nanjing University, China (minghuai.wang@nju.edu.cn) \\ ${ }^{3}$ Institute of Earth Sciences, The Hebrew University of Jerusalem, Jerusalem 91904, Israel (daniel.rosenfeld@huji.ac.il) \\ ${ }^{4}$ School of Atmospheric Sciences, Nanjing University, China (yannianzhu@gmail.com) \\ ${ }^{5}$ Department of Physics, University of Maryland, Baltimore County (UMBC), Baltimore, MD, USA (zzbatmos@umbc.edu)
}

Representing subgrid variability of cloud properties has always been a challenge in global climate models (GCMs). In microphysics schemes, the effects of subgrid cloud variability on warm rain process rates calculated based on mean cloud properties are usually accounted for by scaling process rates by an enhancement factor (EF) that is derived from the subgrid variance of cloud water. In our study, we find that the EF derived from Cloud Layers Unified by Binormals (CLUBB) in Community Earth System Model Version 2 (CESM2) is severely overestimated in most of the oceanic areas, which leads to the strong overestimation in the autoconversion rate. Through an EF formula based on empirical fitting of MODIS, we improve the EF in the liquid phase clouds. Results show that the model has a more reasonable relationship between autoconversion rate, cloud liquid water content (LWC), and droplet number concentration (CDNC) in warm rain simulation. The annual mean liquid cloud fraction (LCF), liquid water path (LWP), and CDNC show obvious increases for marine stratocumulus, where the probability of precipitation (POP) shows an obvious decrease. The annual mean LCF, cloud optical thickness (COT), and shortwave cloud forcing (SWCF) match better with observation. The sensitivity of LWP to aerosol decreases obviously. The sensitivities of LCF, LWP, cloud top droplet effective radius (CER), and COT to aerosol are in better agreement with MODIS, but the model still underestimates the response of cloud albedo to aerosol. These results indicate the importance of representing reasonable subgrid cloud variabilities in the simulation of cloud properties and aerosol-cloud interaction in climate models. 